\title{
SURFACE TERMS ON THE NISHIMORI LINE OF THE GAUSSIAN EDWARDS-ANDERSON MODEL
}

\author{
Pierluigi Contucci ${ }^{\dagger}$, Satoshi Morita ${ }^{\ddagger}$, Hidetoshi Nishimori ${ }^{\ddagger}$ \\ † Dipartimento di Matematica, Università di Bologna, Italy \\ e-mail: contucci@dm.unibo.it \\ $\ddagger$ Department of Physics, Tokyo Institute of Technology, Japan \\ e-mail: smorita@stat.phys.titech.ac.jp
}

\begin{abstract}
For the Edwards-Anderson model we find an integral representation for some surface terms on the Nishimori line. Among the results are expressions for the surface pressure for free and periodic boundary conditions and the adjacency pressure, i.e., the difference between the pressure of a box and the sum of the pressures of adjacent sub-boxes in which the box can been decomposed. We show that all those terms indeed behave proportionally to the surface size and prove the existence in the thermodynamic limit of the adjacency pressure.
\end{abstract}




\section{Introduction}

The next to the leading term in the volume for typical extensive quantities in statistical mechanics like the free energy is usually expected to behave as a surface at least for finite-dimensional (non mean-field) models and regular potentials. This fact has been analyzed since the seminal work by Fisher and Lebowitz [FL] on classical particle systems and followed by a series of results in Euclidean quantum field theories [G, GRS] and in ferromagnetic spin models $[\mathrm{FC}]$.

Much less is known in the spin glass case. In the Edwards-Anderson model for instance the proof that the correction size behavior is indeed a surface came only very recently [CG1, CG2] stemming from the spectacular interpolation technique which changed the landscape of the rigorous results in the mean field spin glass models [GT, G2].

In this paper we perform the analysis of three surface terms for a finite-dimensional model with a non-centered quenched interaction. Our study is made within a generalization of the Nishimori line (see definition in Sect. 2), in which several exact results can be obtained from the gauge symmetries of the model [N, N2] (see also the appendix where we provide a self-contained treatment of some aspects). The first term we consider is the adjacency pressure, i.e., the difference, for free boundary conditions, between the pressure of a box and the sum of the pressures of the disjoint adjacent sub-boxes in which the large box has been decomposed (see $[\mathrm{CF}]$ for its treatment in the ferromagnetic case). We show in Theorem 1 that such a term behaves indeed like the total surface of contact between the sub-boxes and that its value per unit surface exists in the thermodynamic limit. Our method produces in fact an integral representation for its limiting value and is strongly based on an inequality which has been recently proved [MNC] to hold on the NL and which allows to reach in this case the same level of control which one obtains in ferromagnetic models where the second Griffiths inequality [Gr] holds true. To our knowledge this is the only case in disordered systems in which a surface quantity per site can be shown to exist. We consider also two other surface terms: the difference of the

pressure between free and periodic boundary conditions and the pure surface pressure on free boundary conditions. Our method (see Theorems 2 and 3) leads in this case to a 
control on the size of these terms which turns out to be indeed that of the surface of the box.

\section{Surface Terms}

For Ising spins $S_{n}, n \in \Lambda \subset \mathbb{Z}^{d}$, we consider a nearest neighbor potential

$$
U=\sum_{b \in B(\Lambda)} \beta_{b} J_{b} S_{b}
$$

where $B(\Lambda)$ is the set of bonds $b=\left(n, n^{\prime}\right)$, i.e., the couples of nearest neighbor sites $n$ and $n^{\prime}$ in $\Lambda$, the parameter $\beta_{b} \geq 0$ are local inverse temperatures, $S_{b}=S_{n} S_{n^{\prime}}$, and the $J_{b}$ are independent Gaussian random variables defined by the average

$$
\left[J_{b}\right]=\mu_{b} \geq 0
$$

and the variance

$$
\left[\left(J_{b}-\mu_{b}\right)^{2}\right]=\sigma_{b}^{2}
$$

The generalized NL for the present case of local temperature is defined by the two conditions $\beta_{b}=x_{b} / \sigma_{b}$ and $\mu_{b}=\sigma_{b} x_{b}$ for every choice of $x_{b} \geq 0$ [MNC].

By definition the quenched pressure of the model is defined as

$$
P=\int \prod_{b} \frac{d J_{b}}{\sqrt{2 \pi \sigma_{b}^{2}}} e^{-\frac{\left(J_{b}-\mu_{b}\right)^{2}}{2 \sigma_{b}^{2}}} \ln \sum_{\{S\}} e^{\sum_{b} \beta_{b} J_{b} S_{b}}
$$

on the NL one can introduce the Gaussian variable $j_{b}=J_{b} / \sigma_{b}$ whose mean is $x_{b}$ and variance is 1 and observe that the pressure admits the representation

$$
P(\{x\})=\int \prod_{b} \frac{d j_{b}}{\sqrt{2 \pi}} e^{-\frac{\left(j_{b}-x_{b}\right)^{2}}{2}} \ln \sum_{\{S\}} e^{\sum_{b} x_{b} j_{b} S_{b}}
$$

where the notation is stressing the fact that on the NL the only parameters the pressure depends on are the positive numbers $x_{b}$.

The first surface term we want to consider is the difference, at inverse temperature $\beta$, between the pressure of a box $\Lambda$ and the sum of the pressures for a disjoint decomposition 
of it: $\Lambda=\bigcup_{i} \Lambda_{i}$, where $\Lambda$ and $\Lambda_{i}$ are both with free boundaries. We expect such a quantity to be of the size of the corridor $\mathcal{C}$, i.e., the set of bonds joining neighboring regions of the $\Lambda_{i}$ and we will call it $T_{\mathcal{C}}$. To prove it we choose the family $\{x\}$ according to the law

$$
x_{b}= \begin{cases}\beta \sigma \sqrt{t}, & \text { if } b \in \mathcal{C} \\ \beta \sigma, & \text { otherwise }\end{cases}
$$

With this choice the pressure is a function of $\beta$ and $t, P(\beta, t)$. Clearly $P(\beta, 1)=P_{\Lambda}(\beta)$, where $P_{\Lambda}(\beta)$ is the pressure of a box $\Lambda$ at the inverse temperature $\beta$ on the NL and analogously $P(\beta, 0)=\sum_{i} P_{\Lambda_{i}}(\beta)$ since the zero value of the interaction along the corridor makes the subsets independent. By the fundamental theorem of calculus

$$
T_{\mathcal{C}}=P_{\Lambda}(\beta)-\sum_{i} P_{\Lambda_{i}}(\beta)=P(\beta, 1)-P(\beta, 0)=\int_{0}^{1} \frac{\partial}{\partial t} P(\beta, t) d t
$$

Moreover

$$
\frac{\partial}{\partial t} P(\beta, t)=\sum_{b} \frac{\partial P(\{x\})}{\partial x_{b}} \frac{d x_{b}}{d t}
$$

where $x_{b}$ is set according to (2.6) after differential $\partial P / \partial x_{b}$. From (2.6) we obtain

$$
\frac{d x_{b}}{d t}= \begin{cases}\beta \sigma / 2 \sqrt{t}, & \text { if } b \in \mathcal{C} \\ 0, & \text { otherwise }\end{cases}
$$

Introducing the Boltzmann-Gibbs expectation at fixed disorder

$$
\left\langle S_{b}\right\rangle=\frac{\sum_{\{S\}} S_{b} e^{U}}{\sum_{\{S\}} e^{U}}
$$

we can show by a straightforward computation (see appendix) that

$$
\frac{\partial P}{\partial x_{b}}=x_{b}\left[\left\langle S_{b}+1\right\rangle\right]
$$

Putting together $(2.7),(2.8),(2.9)$ and (2.11) we obtain

$$
T_{\mathcal{C}}=\frac{\beta^{2} \sigma^{2}}{2} \sum_{b \in \mathcal{C}} \int_{0}^{1}\left[\left\langle S_{b}+1\right\rangle_{t}\right]_{t} d t
$$

where we have indicated explicitly the dependence on the parameter $t$ of both the Gaussian integration $[\cdots]$ which depends on $t$ through its mean $x_{b}$ and the Boltzmann-Gibbs state 
$\langle\cdots\rangle$ which depends on it through the potential. Let us now write $\beta \sigma=x$. Defining the average bond-spin on the corridor as $S_{\mathcal{C}}=|\mathcal{C}|^{-1} \sum_{b} S_{b}$ we find

$$
T_{\mathcal{C}}=|\mathcal{C}| \frac{x^{2}}{2}\left(1+\int_{0}^{1}\left[\left\langle S_{\mathcal{C}}\right\rangle_{t}\right]_{t} d t\right) .
$$

If, for instance, we choose $\Lambda$ to be a $d$-dimensional square box of side $2 L$ and the sets $\Lambda_{i}$ to be the $2^{d}$ hypercubes which compose it, we immediately find that the cardinality of $\mathcal{C}$ is equal to the surface of $\Lambda$, i.e., $|\mathcal{C}|=d(2 L)^{d-1}$ up to a lower-order term $\left(\mathcal{O}\left(L^{d-2}\right)\right)$. In this case we can write with obvious meaning of the symbols

$$
\frac{T_{L}}{L^{d-1}}=d x^{2} 2^{d-2}\left(1+\int_{0}^{1}\left[\left\langle S_{\mathcal{C}}\right\rangle_{t}\right]_{t} d t\right)
$$

This representation allows us to show that the limit of the quantity on the left-hand side when $L$ goes to $\infty$ does exist. In fact it is possible to see that each correlation $\left[\left\langle S_{b}\right\rangle\right]$ is, on the NL, monotonic increasing with respect to any $x_{b^{\prime}}$ :

$$
\frac{d}{d x_{b^{\prime}}}\left[\left\langle S_{b}\right\rangle\right] \geq 0
$$

This inequality originally proved in $[\mathrm{MNC}]$ (see also the appendix) plays the same role as the second Griffiths inequality for ferromagnetic systems [Gr] and leads immediately, by a similar argument, to the existence of the thermodynamic limit of the correlation functions for free boundary conditions. From the existence of the bond correlation functions it is possible to deduce the existence of the quantity $\left[\left\langle S_{\mathcal{C}}\right\rangle_{t}\right]_{t}$, which turns out to coincide with its thermodynamically relevant component, i.e., the value of the bond correlation function away from the boundary of each cube hyperface. The proof runs in full parallel to the ferromagnetic case and is treated, for instance, in [FC] (see also [Si] Theorem II.8.3).

The arguments developed so far can be summarized as follows:

Theorem 1 On the $N L$ the term $T_{L}$ produced by subtracting from the pressure of a square d-dimensional box of side $2 L$ (with free boundaries) those of the composing sub-boxes of side $L$ grows in proportion to the surface size $L^{d-1}$. The ratio

$$
\frac{T_{L}}{L^{d-1}}
$$


exists in the thermodynamic limit and admits the representation

$$
d x^{2} 2^{d-2}\left(1+\int_{0}^{1}\left[\left\langle S_{1} S_{2}\right\rangle_{t}\right]_{t} d t\right)
$$

where the two spins $S_{1}$ and $S_{2}$ are two nearest neighbors of two adjacent boxes far away from the outer boundaries. The quenched states are computed for free boundary conditions and with the interpolation scheme (2.6).

It is interesting to observe that while the same term is known to have a surface size also in the Edwards-Anderson model with symmetric randomness [CG2] its existence is still an open question due to the lack of an inequality of the type of the second Griffiths inequality which would ensure the existence by monotonicity of the correlation functions.

The next term we want to estimate is the difference between the quenched pressure for periodic $(\Pi)$ and free $(\Phi)$ boundary conditions. In order to find its size dependence we consider the $d$-dimensional torus of side $L, \Pi_{L}$, and we define $\mathcal{C}$ to be the standard cut of the torus, i.e., the set of bonds cutting along which the torus unfolds into the hypercube $\Lambda_{L}$. The argument proceeds like in the previous case with formally the same definition of the interpolating parameters $\{x\}$ as in (2.6). In this case we have

$$
T_{L}^{(\Pi, \Phi)}=P_{\Pi_{L}}(\beta)-P_{\Lambda_{L}}(\beta)=P_{\Pi_{L}}(\beta, 1)-P_{\Pi_{L}}(\beta, 0)=\int_{0}^{1} \frac{\partial}{\partial t} P_{\Pi_{L}}(\beta, t) d t .
$$

Since the cardinality of $\mathcal{C}$ is now $d L^{d-1},(2.13)$ lead to

$$
T_{L}^{(\Pi, \Phi)}=\frac{1}{2} d x^{2} L^{d-1}\left(1+\int_{0}^{1}\left[\left\langle S_{\mathcal{C}}\right\rangle_{t}^{\left(\Pi_{L}\right)}\right]_{t} d t\right)
$$

where the brackets represent the Boltzmann-Gibbs state at fixed disorder for periodic boundary conditions and the quenched states are computed in the suitable interpolation scheme. We can thus formulate the following theorem:

Theorem 2 On the $N L$ the term $T_{L}^{(\Pi, \Phi)}$ obtained by subtracting from the pressure for periodic boundary conditions the one with free boundary conditions grows like a surface and admits the integral representation

$$
\frac{1}{2} d x^{2} L^{d-1}\left(1+\int_{0}^{1}\left[\left\langle S_{\mathcal{C}}\right\rangle_{t}^{\left(\Pi_{L}\right)}\right]_{t} d t\right)
$$


where the quenched states are computed for periodic boundary conditions and with the interpolation scheme (2.6) along the standard cut of the torus.

Although the inequality (2.15) holds in complete generality, one cannot deduce from it the existence by monotonicity of the correlation functions for periodic boundary conditions and, by consequence, the existence result of the surface term in the thermodynamic limit is not within reach at present. One encounters the same difficulty in the ferromagnetic case (see again [Si] Theorem II.8.3).

The final expression we want to consider represents the genuine surface pressure defined, for boundary conditions $\Xi(=\Pi$ or $\Phi)$, as (see $[\mathrm{Si}]$ )

$$
T_{\partial \Lambda}^{(\Xi)}=P_{\Lambda}^{(\Xi)}-p|\Lambda|
$$

where $p$ is the limiting pressure per site. The existence by monotonicity of the quantity $p$ on the NL has been proved for free boundary conditions in $[\mathrm{MNC}]$. The result of the previous theorem tell us that the difference $P_{\Pi_{L}}(\beta)-P_{\Lambda_{L}}(\beta)$ is of the order of a surface: by consequence the pressure per site $p_{L}=P_{\Lambda}^{(\Xi)} / L^{d}$ converges to the same value $p$ for both free and periodic boundary conditions. One may prove along the same lines (using interpolation between two assigned boundary conditions as in (2.6) and taking the fact into account that (2.11) is valid for any boundaries) that the limiting pressure exists for a wide class of boundary conditions and its value is the same for all of them.

Let us take a square box $\Lambda$ of side $L$ and its $k$-magnification, i.e., the box of side $k L$. For free boundary conditions

$$
P_{L}^{(\Phi)}=\left[\ln Z_{L}^{(\Phi)}\right]=k^{-d}\left[\ln \left(Z_{L}^{(\Phi)}\right)^{k^{d}}\right]
$$

On the other hand the same limiting pressure can be obtained for instance for periodic boundary conditions

$$
p L^{d}=\lim _{k \rightarrow \infty} k^{-d}\left[\ln Z_{k L}^{(\Pi)}\right]
$$

By (2.22) and (2.23) we obtain

$$
\begin{aligned}
T_{\partial \Lambda}^{(\Phi)} & =\left(P_{L}^{(\Phi)}-p L^{d}\right) \\
& =\lim _{k \rightarrow \infty} k^{-d}\left[\ln \left(Z_{L}^{(\Phi)}\right)^{k^{d}}-\ln Z_{k L}^{(\Pi)}\right]
\end{aligned}
$$


In this case we define the corridor $\mathcal{C}$ as the set of nearest neighbors belonging to adjacent boxes of side $L$ which compose the large torus of side $k L$. Following the same procedure as before we arrive at

$$
T_{\partial \Lambda}^{(\Phi)}=-\lim _{k \rightarrow \infty} k^{-d} \int_{0}^{1} \frac{\partial}{\partial t} P_{\Pi_{k L}}(\beta, t) d t
$$

in this case the cardinality of $\mathcal{C}$ turns out to be $d L^{d-1} k^{d}$ which, together with the computation of the derivative, leads us to the following result:

Theorem 3 On the NL the surface pressure for free boundary conditions grows like a surface and admits the integral representation

$$
T_{\partial \Lambda}^{(\Phi)}=-\frac{d}{2} x^{2} L^{d-1}\left(1+\int_{0}^{1}\left[\left\langle S_{\mathcal{C}}\right\rangle_{t}^{\left(\Pi_{\infty L}\right)}\right]_{t} d t\right)
$$

where the brackets represent the Boltzmann-Gibbs state of an infinite system for periodic boundary conditions made up of boxes of side $L$ and interpolated along the adjacent bonds. Since $T_{L}^{(\Pi, \Phi)}=T_{\partial \Lambda}^{(\Pi)}-T_{\partial \Lambda}^{(\Phi)}$, we may use Theorem 2 and (2.26) to obtain

$$
T_{\partial \Lambda}^{(\Pi)}=\frac{1}{2} d x^{2} L^{d-1}\left(\int_{0}^{1}\left[\left\langle S_{\mathcal{C}}\right\rangle_{t}^{\left(\Pi_{L}\right)}\right]_{t} d t-\int_{0}^{1}\left[\left\langle S_{\mathcal{C}}\right\rangle_{t}^{\left(\Pi_{\infty L}\right)}\right]_{t} d t\right) .
$$

It is interesting to observe that on the NL of the Edwards-Anderson model the surface

pressure fulfils the inequalities $T_{\partial \Lambda}^{(\Phi)} \leq 0$ which is the same as in the ferromagnetic case. This fact is totally non trivial a priori because the interactions have no definite sign in our model and the Griffiths inequalities do not hold in the standard form; nevertheless the positivity of the average of the interaction is enough to guarantee that the sign of the surface pressure persists in the disordered case.

\section{Conclusion}

In this paper, we have investigated some surface terms on the NL for the EdwardsAnderson model: the surface pressure for free and periodic boundary conditions, the difference of the pressure between these boundary conditions and the adjacency pressure, which is defined as the difference between the pressure of the box and the sum of 
the pressure for its disjoint decomposition. Each of them has been shown to have an integral representation which is proportional to the surface size. Moreover we showed that the adjacency pressure has a thermodynamic limit. These results are similar to the ferromagnetic case, but is quite non-trivial in disordered systems.

\section{Appendix}

In this appendix, we prove the Griffiths inequalities for the Gaussian Edwards-Anderson model on the NL following $[\mathrm{MNC}]$ :

$$
\begin{gathered}
\frac{d P}{d x_{b}}=x_{b}\left[\left\langle S_{b}+1\right\rangle\right] \geq 0, \\
\frac{d}{d x_{b^{\prime}}}\left[\left\langle S_{b}\right\rangle\right]=2 x_{b^{\prime}}\left[\left(\left\langle S_{b} S_{b^{\prime}}\right\rangle-\left\langle S_{b}\right\rangle\left\langle S_{b^{\prime}}\right\rangle\right)^{2}\right] \geq 0 .
\end{gathered}
$$

Before the proof of these inequalities, we prove the following identities which hold on the NL:

$$
\begin{gathered}
{\left[\left\langle j_{b} S_{b}\right\rangle\right]=x_{b},} \\
{\left[\left\langle S_{b}\right\rangle\right]=\left[\left\langle S_{b}\right\rangle^{2}\right] .}
\end{gathered}
$$

To obtain the first identity, let us consider the average of the Gaussian random variable $j_{b}$

$$
\left[j_{b}\right]=\int \prod_{b^{\prime}} \frac{d j_{b^{\prime}}}{\sqrt{2 \pi}} e^{-\frac{\left(j_{b^{\prime}}-x_{b^{\prime}}\right)^{2}}{2}} j_{b} .
$$

The right-hand side does not change under a local transformation $j_{b} \rightarrow j_{b} \sigma_{b}$, where $\sigma_{b}$ (to be distinguished from $\sigma_{b}$ in $(2.3)$ ) is a product of two Ising spins at the edge of the bond $b=\left(n, n^{\prime}\right)$, that is, $\sigma_{b}=\sigma_{n} \sigma_{n^{\prime}}$. Thus it can be rewritten using the nearest neighbor potential (2.1),

$$
\left[j_{b}\right]=\int \prod_{b^{\prime}} \frac{d j_{b^{\prime}}}{\sqrt{2 \pi}} e^{-\frac{j_{b^{\prime}}^{2}+x_{b^{\prime}}^{2}}{2}} e^{U} j_{b} \sigma_{b} .
$$

Since the value of $\sigma_{n}$ is arbitrary, we can sum this equation over all possible values of $\left\{\sigma_{n}\right\}$ and divide the result by $2^{N}$ ( $N$ is the number of spins). We then obtain using the Boltzmann-Gibbs expectation (2.10),

$$
\left[j_{b}\right]=\int \prod_{b^{\prime}} \frac{d j_{b^{\prime}}}{\sqrt{2 \pi}} e^{-\frac{j_{b^{\prime}}^{2}+x_{b^{\prime}}^{2}}{2}} \sum_{\{\sigma\}} e^{U}\left\langle j_{b} S_{b}\right\rangle,
$$


where we have written $S_{n}$ for $\sigma_{n}$. The right-hand side of the above equation is equal to $\left[\left\langle j_{b} S_{b}\right\rangle\right]$. This fact can be confirmed by applying the local transformation to $\left[\left\langle j_{b} S_{b}\right\rangle\right]$. We note that the expectation $\left\langle j_{b} S_{b}\right\rangle$ does not change under this transformation because the transformation for spins, $S_{n} \rightarrow S_{n} \sigma_{n}$ cancels with the one for the Gaussian random variables. Consequently, we obtain (4.30) because the mean of the Gaussian random variable $j_{b}$ is $x_{b}$.

The second identity (4.31) is obtained similarly to the first one: It holds because the expectation $\left\langle S_{b}\right\rangle$ changes to $\sigma_{b}\left\langle S_{b}\right\rangle$ under the transformation for the Gaussian random variables and $\left\langle S_{b}\right\rangle^{2}$ does not change.

Now, to prove the first inequality (4.28), we consider the total derivative with respect to $x_{b}$. The pressure function depends on $x_{b}$ through the Gaussian distribution and the nearest neighbor potential. The derivative of the Gaussian distribution is rewritten as follows:

$$
\begin{aligned}
\int \frac{d j_{b}}{\sqrt{2 \pi}} \frac{d}{d x_{b}}\left(e^{-\frac{\left(j_{b}-x_{b}\right)^{2}}{2}}\right) f\left(j_{b}\right) & =-\int \frac{d j_{b}}{\sqrt{2 \pi}} \frac{d}{d j_{b}}\left(e^{-\frac{\left(j_{b}-x_{b}\right)^{2}}{2}}\right) f\left(j_{b}\right) \\
& =\left[\frac{d}{d j_{b}} f\left(j_{b}\right)\right]
\end{aligned}
$$

where we used integration by parts to obtain the final line. Thus we find

$$
\frac{d P}{d x_{b}}=\int \prod_{b^{\prime}} \frac{d j_{b^{\prime}}}{\sqrt{2 \pi}} e^{-\frac{\left(j_{b^{\prime}}-x_{b^{\prime}}\right)^{2}}{2}}\left(\frac{\partial}{\partial j_{b}}+\frac{\partial}{\partial x_{b}}\right) \ln \sum_{\{S\}} e^{\sum_{b} \beta_{b} j_{b} S_{b}}
$$

Since a straightforward calculation yields

$$
\left(\frac{\partial}{\partial j_{b}}+\frac{\partial}{\partial x_{b}}\right) \ln \sum_{\{S\}} e^{\sum_{b} \beta_{b} j_{b} S_{b}}=x_{b}\left\langle S_{b}\right\rangle+\left\langle j_{b} S_{b}\right\rangle,
$$

we finally obtain from $(4.30)$

$$
\frac{d P}{d x_{b}}=x_{b}\left[\left\langle S_{b}\right\rangle\right]+\left[\left\langle j_{b} S_{b}\right\rangle\right]=x_{b}\left[\left\langle S_{b}+1\right\rangle\right] \geq 0,
$$

which is the first inequality (4.28).

The proof of the second inequality (4.29) is a little more complicated than that of the first one. The derivative by $x_{b}$ is calculated similarly to (4.35) to obtain

$$
\frac{d}{d x_{b^{\prime}}}\left[\left\langle S_{b}\right\rangle\right]=\left[\left(x_{b^{\prime}}+j_{b^{\prime}}\right)\left(\left\langle S_{b} S_{b^{\prime}}\right\rangle-\left\langle S_{b}\right\rangle\left\langle S_{b^{\prime}}\right\rangle\right)\right]
$$


We can eliminate the Gaussian random variable $j_{b^{\prime}}$ using the following property of the Gaussian distribution:

$$
\left[\left(j_{b}-x_{b}\right) f\left(j_{b}\right)\right]=-\int \frac{d j_{b}}{\sqrt{2 \pi}} \frac{d}{d j_{b}}\left(e^{-\frac{\left(j_{b}-x_{b}\right)^{2}}{2}}\right) f\left(j_{b}\right)=\left[\frac{d}{d j_{b}} f\left(j_{b}\right)\right] .
$$

Thus, we obtain

$$
\begin{aligned}
\frac{d}{d x_{b^{\prime}}}\left[\left\langle S_{b}\right\rangle\right] & =2 x_{b^{\prime}}\left[\left\langle S_{b} S_{b^{\prime}}\right\rangle-\left\langle S_{b}\right\rangle\left\langle S_{b^{\prime}}\right\rangle\right]+\left[\frac{\partial}{\partial j_{b^{\prime}}}\left(\left\langle S_{b} S_{b^{\prime}}\right\rangle-\left\langle S_{b}\right\rangle\left\langle S_{b^{\prime}}\right\rangle\right)\right] \\
& =2 x_{b^{\prime}}\left[\left\langle S_{b} S_{b^{\prime}}\right\rangle-\left\langle S_{b}\right\rangle\left\langle S_{b^{\prime}}\right\rangle-\left\langle S_{b} S_{b^{\prime}}\right\rangle\left\langle S_{b^{\prime}}\right\rangle+\left\langle S_{b}\right\rangle\left\langle S_{b^{\prime}}\right\rangle^{2}\right] .
\end{aligned}
$$

Since the following set of identities can be proved similarly to (4.31):

$$
\begin{gathered}
{\left[\left\langle S_{b} S_{b^{\prime}}\right\rangle\right]=\left[\left\langle S_{b} S_{b^{\prime}}\right\rangle^{2}\right]} \\
{\left[\left\langle S_{b}\right\rangle\left\langle S_{b^{\prime}}\right\rangle\right]=\left[\left\langle S_{b} S_{b^{\prime}}\right\rangle\left\langle S_{b^{\prime}}\right\rangle\right]=\left[\left\langle S_{b}\right\rangle\left\langle S_{b^{\prime}}\right\rangle\left\langle S_{b} S_{b^{\prime}}\right\rangle\right]} \\
{\left[\left\langle S_{b}\right\rangle\left\langle S_{b^{\prime}}\right\rangle^{2}\right]=\left[\left\langle S_{b}\right\rangle^{2}\left\langle S_{b^{\prime}}\right\rangle^{2}\right],}
\end{gathered}
$$

we find

$$
\frac{d}{d x_{b^{\prime}}}\left[\left\langle S_{b}\right\rangle\right]=2 x_{b^{\prime}}\left[\left(\left\langle S_{b} S_{b^{\prime}}\right\rangle-\left\langle S_{b}\right\rangle\left\langle S_{b^{\prime}}\right\rangle\right)^{2}\right] \geq 0
$$

and the second inequality (4.29) has been proved.

\section{Acknowledgments.}

One of us (P.C.) thanks Sandro Graffi for introducing him to the surface pressure problems and the Tokyo Institute of Technology for the warm hospitality and the stimulating atmosphere during the visit in which this work was done. This work was supported by the Grant-in-Aid for Scientific Research on Priority Area "Statistical-Mechanical Approach to Probabilistic Information Processing" by the MEXT. P.C. was partially supported by University of Bologna, Funds for Selected Research Topics and Funds for Agreement with Foreign Universities.

\section{References}

[FL] M. E. Fisher and J. L. Lebowitz, Commun. Math. Phys., 19, 251-272, (1970). 
[G] F. Guerra, Phys. Rev. Lett., 28, 1213-1215, (1972)

[GRS] F. Guerra, L. Rosen and B. Simon, Ann. Inst. H. Poincare, A25, 231-334, (1976).

[FC] M. E. Fisher and G. Caginalp, Commun. Math. Phys., 56, 11-56, (1977)

[CG1] P. Contucci and S. Graffi, Jou. Stat. Phys., 115, 581-589,(2004)

[CG2] P. Contucci and S. Graffi, Commun. Math. Phys., 248, 207-216, (2004)

[GT] F. Guerra and F. Toninelli, Commun. Math. Phys., 230, 71-79 (2002)

[G2] F. Guerra, Commun. Math. Phys., 233, 1-12 (2003)

[N] H. Nishimori, Prog. Theor., 66 1169-1181 (1981)

[N2] H. Nishimori, Statistical Physics of Spin Glasses and Information Processing: An Introduction, Oxford: Oxford University Press, (2001)

[CF] G. Caginalp and M. E. Fisher, Commun. Math. Phys., 65, 247-280, (1979)

[MNC] S. Morita, H. Nishimori and P. Contucci, Jou. Phys. A: Math. and Gen., 37, L203-L209, (2004)

[Gr] R. B. Griffiths, Jou. Math. Phys., 8, 478-483, 484-489, (1967)

[Si] B. Simon, The statistical mechanics of lattice gases, Princeton Univ. Press, (1992) 